\title{
Acute compartment syndrome of the forearm and hand in a patient of spine surgery -A case report-
}

\author{
Jung-Ah Lee, Yeon Soo Jeon, Hong Soo Jung, Hyung-Gun Kim, and Yong Shin Kim \\ Department of Anesthesiology and Pain Medicine, St. Vincent Hospital, Catholic University of Korea, Suwon, Korea
}

\begin{abstract}
A 38-year-old woman underwent a 4-hour operation in the prone position for a laminectomy at C4-7 and posterior cervical decompressive fusion at C7-T1 under general anesthesia. After undraping at the end of surgery, considerable swelling with many blisters of the left forearm and hand was observed. The chest roll at the left side had moved cephalad into the axilla and compressed the axillary structures. An emergency fasciotomy to decompress the compartments of the forearm and dorsal surface of the hand was performed. In the post anesthesia care unit, the radial pulse of the left hand was palpable and the level of oxygen saturation was normal. Forearm and hand edema subsided gradually over several days and the patient was discharged with full function of her left arm. This compartment syndrome suggests careful attention should be paid to the position of the chest roll when the prone position is established for a long duration. (Korean J Anesthesiol 2010; 59: 53-55)
\end{abstract}

Key Words: Axilla, Compartment syndrome, Prone position.

Intraoperative compartment syndrome is reported infrequently but is a potentially devastating perioperative complication that typically involves the legs or arms [1]. Most surgical patients have this problem while being anesthetized for a long duration and positioned in lithotomy or lateral decubitus [2].

We recently encountered compartment syndrome in a patient in the prone position. This report describes a case of forearm and hand compartment syndrome resulting from compression of the axillary structure due to a displaced chest roll in a patient in the prone position during spine surgery.

\section{Case Report}

A 38-year-old, $160 \mathrm{~cm}, 60 \mathrm{~kg}$ woman, American society of anesthesiologist physical status 1 , with quadriparesis from ossification of the posterior longitudinal ligament at C4-T1 and myelopathy was scheduled for a laminectomy at C4-7 and posterior cervical decompressive fusion at $\mathrm{C} 7-\mathrm{T} 1$. Her previous medical history was unremarkable. The routine monitors including an electrocardiogram, noninvasive blood pressure (NIBP), and pulse oxymetry were used. General anesthesia was

Received: September 16, 2009. Revised: 1st, October 1, 2009; 2nd, October 14, 2009. Accepted: October 23, 2009.

Corresponding author: Yong Shin Kim, M.D., Ph.D., Department of Anesthesiology and Pain Medicine, St. Vincent Hospital, Catholic University of Korea, 93, Ji-dong, Paldal-gu, Suwon 440-060, Korea. Tel: 82-31-249-7214, Fax: 82-31-258-4212, E-mail: aneskim@catholic.ac.kr @ This is an open-access article distributed under the terms of the Creative Commons Attribution Non-Commercial License (http:// creativecommons.org/licenses/by-nc/3.0/), which permits unrestricted non-commercial use, distribution, and reproduction in any medium, provided the original work is properly cited. 
induced with intravenous $1 \%$ lidocaine $40 \mathrm{mg}$, propofol $120 \mathrm{mg}$ and rocuronium $50 \mathrm{mg}$ via a preexisting 18-gauge IV catheter in the vein on the left antecubital area without difficulty. The trachea was intubated, and ventilation was controlled. General anesthesia was maintained with 6 vol\% desflurane and 50\% $\mathrm{N}_{2} \mathrm{O}$ in oxygen. A right radial artery catheter was inserted successfully to allow continuous monitoring of the arterial blood pressure. A 16-gauge catheter was also placed in a large vein on the dorsal surface of the left foot. It was confirmed that both intravenous catheters functioned well. The patient was then placed in the prone position on the operating table. The patient's arm was tucked at her sides with her palms facing inward against the lateral thighs under the draw sheet.

To prevent a risk of chest compression, two chest rolls were placed under the both lateral chest walls. The placement of chest rolls was checked to confirm that they had been placed correctly under the medial wall of the axilla. No surgical bolster or padding was applied under the axilla because her arms had been tucked to her side. The NIBP was measured using an automated oscillatory blood pressure cuff on the left upper arm every 20 minutes and a pulse oximeter probe was placed on the right thumb. All medication was injected through the IV catheter on the left foot, not into the left arm. The patient received $700 \mathrm{ml}$ of a crystalloid solution through the left arm IV catheter. Nothing unusual was noted intraoperatively. However, the area of the left arm covered with drapes was unavailable for a direct inspection. The operation lasted for 4 hours.

After undraping at the end of surgery, the left forearm and hand of the patient were found to be quite swollen with many blisters (Fig. 1). The left upper extremity was very cool and cyanotic, and the radial pulses were not palpable well. The pulse oximeter did not detect any waveforms on the left hand. Digital capillary refill was found to be delayed compared to

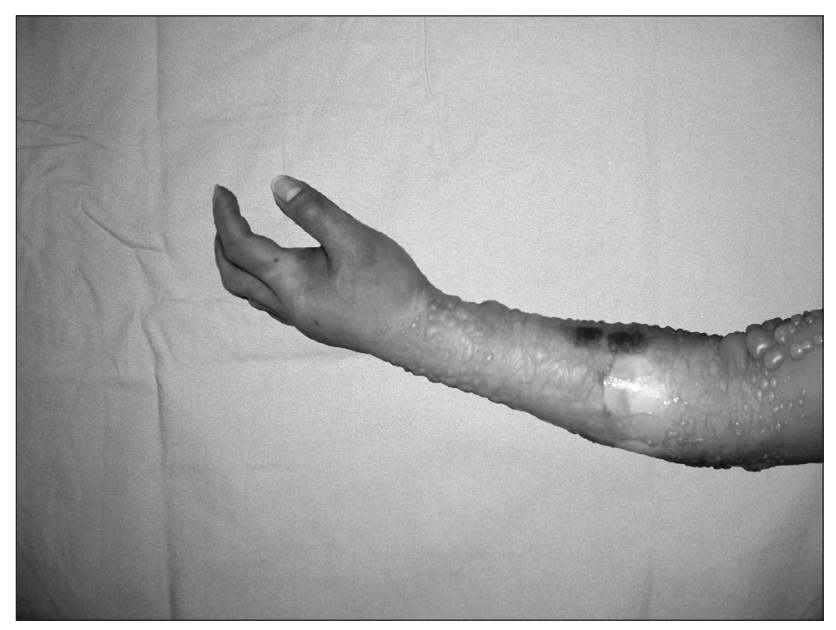

Fig. 1. Swollen left forearm and hand with many blisters. the contralateral extremity. The NIBP cuff and left antecubital catheter were removed. A hand surgeon was rapidly consulted and a fasciotomy was performed on the forearm and dorsal surface of the hand to reduce the increased pressure of the compartments. A large amount of fluid, which was likely to be an extravasated crystalloid solution, was drained when the surgeon squeezed the patient's arm during the fasciotomy.

The patient was extubated in the operating room. In the post anesthesia care unit, a physical examination revealed palpable radial arterial pulses and normal capillary refilling. The pulse oximeter probe successfully detected a waveform and an arterial saturation appeared to be $100 \%$. In the intensive care unit, edema of the forearm and hand gradually subsided over several days. She was discharged with full function of her left arm.

\section{Discussion}

Compartment syndrome is defined as a condition in which the circulation and function of the tissues within a closed space are compromised by increased pressure within that space [3]. Compartment syndromes related to anesthesia have been reported to occur with drug extravasation $[4,5]$, pressurized IV fluids [6], hypertonic saline [7], attempts at cannulating arteries [8,9], and intraarterial barbiturates [10]. Regardless of the etiology, the pathophysiology is an increasing intracompartmental pressure that hinders microcirculatory perfusion, which can result in ischemic injury to the muscle, nerves and other compressed structures.

The most common postoperative compartment syndrome is posterior compartment syndrome of the leg from direct pressure of the calf muscles due to the lithotomy position [11]. However, compartment syndrome of the forearm is being increasingly recognized $[4,5,8,9]$.

A diagnosis of compartment syndrome is based on evidence of tissue ischemia, such as pain, pallor, diminished sensation, motor weakness in addition to compartment swelling and tightness on the examination. A definitive diagnosis is made after direct measurements of the intracompartmental pressure. A diagnosis of compartment syndrome is difficult in an anesthetized patient, in which the early signs and symptoms can be masked by the anesthesia. Therefore, intraoperative vigilance and ongoing patient assessment by the anesthesiologist is essential for making a timely diagnosis and minimizing the risk of permanent injury. Monitoring of the upper extremities with an intraarterial pressure wave and pulse oximetry can lead to the earlier detection of an increased compartmental pressure.

Once the syndrome is recognized, an immediate fasciotomy to decompress the neurovascular compartment is essential to prevent further ischemia and decrease the level of nerve 
dysfunction. A fasciotomy will allow acutely edematous muscles to bulge through the divided fascia, which will relieve the pressure on the neurovascular bundle. Increases in compartment pressure lasting longer than 12 hours are associated with chronic functional defects, including sensory changes, motor weakness and eventual contractures [12]. Accordingly, prompt recognition of compartment syndrome in the immediate postoperative period is essential for preventing muscle and nerve necrosis and, ultimately, severe disability.

In this case, there were several factors that adversely affect the local tissue blood flow and perfusion and predispose the patient to compartment syndrome of the forearm. This study suggests that the chest roll at the left side, placed under the medial wall of the axilla, had moved cephalad into the axilla and compressed the axillary structures. In addition, the blood pressure cuff had been applied to the left arm and the noninvasive blood pressure was measured repeatedly using an automated oscillatory blood pressure cuff. This also could pose a risk of prolonging the partial venous obstruction and possibly even reduce the arterial blood flow in the left arm. In addition, a maintenance crystalloid solution was administered through the left antecubital vein. Extravasation of the fluids may be one of the causes resulting in compartment syndrome in this patient because an increase in pressure within a limited space restricts the circulation to the area leading to ischemia. Consequently, the significantly swollen cyanotic forearm with many blisters in this patient was caused by a displaced chest roll, repeated cuff pressure and extravasation of fluid.

This type of syndrome might conceivably arise in the operating room where the patient is lying in deep anesthesia for a prolonged period. Therefore, care for safe positioning during anesthesia is essential to avoid these pressure problems. A frequent assessment of an extremity with an indwelling IV catheter should be made by the anesthesiologist because perioperative compartment syndrome can occur in conventional conditions of care. In addition, it is recommended that fluid be infused in an observed intravenous site if possible.

In conclusion, a displaced chest roll for a prolonged procedure in the prone position may result in compartment syndrome of the upper extremity, which can be complicated by the administration of IV fluid in the same side, even in an uncomplicated patient. Surgeons and anesthesiologists need to be alert to the possibility of a compartment syndrome, particularly when the patient is unable to communicate with the medical team.

\section{References}

1. Martin JT. Compartment syndrome: concenpts and perspective for the anesthesiologist. Anesth Analg 1992; 75: 275-83.

2. Warner ME, LaMaster LM, Thoeming AK, Marienau ME, Warner MA. Compartment syndrome in surgical patients. Anesthesiology 2001; 94: 705-8.

3. Matsen FA 3rd. Compartmental syndrome: a unified concept. Clin Orthop Relat Res 1975; 113: 8-14.

4. Edward JJ, Samuels D, Fu ES. Forearm compartment syndrome from intravenous mannitol extravasation during general anesthesia. Anesth Analg 2003; 96: 245-6.

5. Bortoluxxi ME, Hunter JG, Handal AG. Forearm compartment syndrome after diazepam administration. Anesthesiology 1991; 75: 159-60.

6. Willsey DB, Peterfreund RA. Compartment syndrome of the upper arm after pressurized infiltration of intravenous fluid. J Clin Anesth 1997; 9: 428-30.

7. Mabee JR, Bostwick TL, Burke MK. Iatrogenic compartment syndrome from hypertonic saline injection in Bier block. J Emerg Med 1994; 12: 473-6.

8. Qvist J, Peterfreund RA, Perlmutter GS. Transient compartment syndrome of the forearm after attempted radial artery cannulation. Anesth Analg 1996; 83: 183-5.

9. Horlocker TT, Bishop AT. Compartment syndrome of the forearm and hand after brachial artery cannulation. Anesth Analg 1995; 81: 1092-4.

10. Morgan NR, Waugh TR, Boback MD. Volkmann's ischemic contracture after intra-arterial injection of secobarbital. JAMA 1970; 212: 476-8.

11. Lydon JC, Spielman FJ. Bilateral compartment syndrome following prolonged surgery in the lithotomy position. Anesthesiology 1984; 60: $236-8$

12. Matsen FA 3rd, Clawson DK. The deep posterior compartmental syndrome of the leg. J Bone Joint Surg 1975; 57: 34-9. 